\title{
Significant Decrease in Seasonal Influenza in the COVID-19 Era: Impact of Global Movement Restrictions?
}

\author{
Takuma Hayashia, b, d, Ikuo Konishia, c
}

\section{To the Editor}

\section{Decrease of seasonal influenza in COVID-19 era}

In early June 2020, the first wave of the COVID-19 pandemic ended in many countries. What followed were the strictest historical migration and travel blockades implemented worldwide. At that time, healthcare professionals witnessed the sudden early end of the 2019 - 2020 seasonal flu season in the northern hemisphere.

According to medical and public health experts, the significant decrease in those presenting for influenza testing may have affected the eventual count of those infected with seasonal influenza in 2019 - 2020. The significant decline in seasonal flu counts in 2019 - 2020 is also due to the same infection precautions as those recommended to prevent COVID-19 infection.

During the COVID-19 epidemic setting in the United States (US), the number of people infected with the influenza virus this season has plummeted by $98 \%$ compared to an average year. This is tempered by a $61 \%$ reduction in flu sample analysis volumes [1]. The US Centers for Disease Control and Prevention rated the recent flu season "moderate" despite an estimated incidence of 38 million illnesses and the death of 22,000 individuals. Compared to the average year, the number of seasonal influenza infections and deaths is significantly lower [1].

The numbers infected with seasonal influenza, often feared as a simultaneous epidemic with COVID-19, have been reported in Japan. The number of cases reported in the week to December 13, 2020 is 57 nationwide (counted across 5,000 institutions). Compared to historical averages for Japan, the 2020 - 2021 infection counts continue to be significantly

Manuscript submitted February 12, 2021, accepted March 4, 2021

Published online March 19, 2021

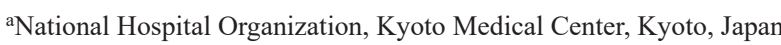

bJapan Science and Technology Agency (JST) START Program, Tokyo, Japan 'Kyoto University Graduate School of Medicine, Kyoto, Japan

${ }^{\mathrm{d} C o r r e s p o n d i n g ~ A u t h o r: ~ T a k u m a ~ H a y a s h i, ~ N a t i o n a l ~ H o s p i t a l ~ O r g a n i z a t i o n, ~}$ Kyoto Medical Center, 1-1 Fukakusa Mukaihata-Cho, Fushimi-Ku, Kyotocity, Kyoto 612-8555, Japan. Email: yoyoyo224@hotmail.com

doi: https://doi.org/10.14740/jocmr4450 lower. According to a report by the National Institute of Infectious Diseases in Japan, the signs of the epidemic of seasonal influenza in Japan have not yet been recognized. The Japanese government is calling on the Japanese people to be aware of the upcoming seasonal influenza epidemic.

In the case of seasonal influenza infection, if the number of patients per week per medical institution exceeds 1 , it is judged an epidemic. Fortunately the fixed-point prevalence of infections is 0.01 . This same report indicated that the number of people infected with seasonal influenza is more than 20,000 at the same time of any other flu season year. The number of people infected with seasonal influenza in 2020 - 2021 continues to be significantly lower.

After the early end of the season of seasonal influenza in the northern hemisphere, there was no epidemic of 2020 seasonal influenza in the southern hemisphere. In the southern hemisphere from April to July 2020, influenza case counts remained extremely low. In Australia, Chile and South Africa, of more than 83,000 samples for seasonal influenza, only 51 people tested positive [1].

Some countries in South America have inconsistent and/or ineffective public health policies directed against COVID-19 (Note 1). Despite what appears to be a general challenge for seasonal and/or epidemic illnesses, the number of people infected with seasonal influenza is extremely small (Note 1). In other words, the absence of people infected with seasonal influenza is not solely due to wearing masks, washing hands and social distance as a countermeasure against the COVID-19 pandemic.

\section{Origin and life cycle of the seasonal influenza virus as a zoonotic disease}

The seasonal influenza virus lurks in frozen lakes and swamps near the Arctic Circle, such as Siberia, Alaska and Canada. Each year in the spring when migratory waterfowl, such as ducks, geese and swans, return to lakes and swamps in Siberia, Alaska and Canada for breeding, the seasonal influenza virus invades the birds and propagates in the intestinal tract [2]. Twice a year, migratory birds spread that acquired seasonal influenza virus via feces on their way to breeding and wintering areas. As many migratory birds travel long distances from the Arctic Circle to the Antarctic Circle, they spread the seasonal influenza virus on a global scale [2].

If the toxicity of the seasonal influenza virus is much 
stronger than the biological defense capability of migratory birds, seasonal influenza virus kills the host. As a result, they collapse together, and neither the seasonal influenza virus nor migratory birds can leave offspring. Therefore, seasonal influenza virus and migratory birds must aim for peaceful coexistence. Pigs are an additional, under-recognized and important vehicle for the seasonal influenza virus. The respiratory cells of pigs are structured, so that many types of viruses may infect them. Virus in waterfowl may be transmitted zoonotically to the respiratory tract of the pig, and in that living body of the pig, influenza virus can and often does mutate. In this process, a seasonal influenza virus variant that can infect humans is born. Pigs are considered as "manufacturing plant" of the new influenza virus.

Regions believed to be an origin of the pandemic of the latest influenza virus are Southeast Asian countries, such as southern China and Vietnam, etc. [3]. Unlike Japan, in southern China and Southeast Asian countries, a single farmer raises multiple types of animals, including pigs, ducks, chickens and cows. When people venture into or reside in rural areas, pigs and ducks are everywhere, and the distance between each animal and between those animals and humans is close. Domesticated ducks and geese are easily infected with the influenza virus transmitted by other waterfowl species [3]. It is widely thought that influenza viruses from ducks, geese and swans infect pigs and are transmitted to humans [4].

In such environments, new influenza viruses are extremely likely. Many of the global outbreaks of seasonal influenza over the last 100 years are believed to have originated in southern China. Even if infection with the new influenza virus is recognized in southern China and Southeast Asian countries, they do not rise to global pandemics if they remain local.

\section{Close relationship between the spread of viral infections and the global movement of people}

It is said that "infectious diseases have no borders". Today, enabled by high-speed mass transportation, nearly 1 billion tourists are in motion annually, according to the statistics of the United Nations in January 2018. In 2019, the number of foreign tourists visiting Japan was 10 million or more. Infectious diseases acquired overseas are increasing in Japan. In recent years, there have been cases of emerging infectious diseases "swine flu, SARS, MERS, H1N1, Flu" appearing and spreading all over the world in a short time.

On a 1-month trip to a developing country, survey results have been reported for cases suffering from various infectious diseases. As the most common infectious disease after return to Japan from foreign travel, diarrhea is observed as $10-30 \%$ of the total infectious diseases $[5,6]$. The next most common ailments acquired during foreign travel are respiratory infections, such as acute upper respiratory tract inflammation. If a returnee from abroad has a fever, it is likely that the returnee has an important infectious disease that should not be overlooked. Possible life-threatening infectious diseases, such as malaria, dengue fever, typhoid fever, rickettsia-related infectious diseases and leptospirosis, are considered serious poten- tial causes of fever.

The migration of people and long-term global cycle of seasonal influenza virus create a serious and regrettably reliable epidemic of seasonal influenza virus. Since at least the 1800 s, the question of whether the seasonal influenza virus has moved internationally or has survived locally among different epidemics has been interesting [7, 8]. Researchers have proposed various hypotheses to understand the global movement of the virus, including the movement of the virus back and forth between temperate regions of the northern and southern hemispheres due to different winter timings. Researchers should also consider the continuous dissemination of epidemics in tropical to temperate regions and dissemination of viruses from China. The early hypotheses of China as the source of COVID-19 are partially explained by the historical importance of China as a source of pandemic viruses and substantially large population of China.

Recently, it has been revealed that the global circulation pattern of seasonal influenza changes with the rate of antigen evolution of various influenza types and subtypes 9. Unlike the A/H3N2 virus, which spreads globally from East-Southeast Asia and India, each year the A/H1N1 and B influenza viruses persist during resolving epidemics in multiple parts of the world [3,9]. In addition, these viruses cause multiple influenza co-occurring gene lines. Occasionally, these co-occurring strains give rise to different antigenic variants and are resistant to influenza vaccines. Therefore, in order to produce a vaccine against influenza virus, researchers must elucidate the biological properties of region-specific influenza B. The vaccine produced during any given year contains three strains of influenza that scientists believe will dominate during that year's flu season.

Previous studies have revealed that humans and their global movement behaviors are key in the flow and circulation of new virus strains. It is natural to think that the suppression of global movement of people will also suppress the global movement of seasonal influenza. Currently, with the spread of COVID-19 infection, various countries are tightening required quarantine time frames (i.e., 10 - 14 days) at the time of entry and exit (submission of health declarations, isolation measures) and are severely limiting immigration flows and VISA issuances. International airline flights are suspended or reduced according to national affairs or public health priorities [10]. Due to the fluid nature of each country's response to COVID-19 infection prevention, consistency cannot be reasonably expected and immigration restrictions are expected to be enforced without notice [10].

Common preventive measures against seasonal influenza are daily routine precautions and influenza vaccination $[11,12]$ (Note 2). By the World Health Organization (WHO), in order to prevent the seasonal influenza infection, wearing the mask and gargle are not recommended (Note 2). Wearing a mask is considered as a means of keeping moisture and preventing the spread of seasonal influenza infection from an infected person significantly. Theoretically, droplets containing the virus would be caught in the stitches of the mask, but sufficient clinical results are required.

Although it has been said that gargling is effective as a preventive measure against infection with the seasonal influenza 
virus, the preventive awareness poster created by the Japanese Ministry of Health, Labor, and Welfare does not include the word "gargle" $[13,14]$. In addition, the official website and media reports of the Japanese Prime Minister's Office have shown that the protective effect of gargling against infection with the seasonal influenza virus has not been scientifically proven $[15,16]$.

Many people wear masks to prevent COVID-19 infection. Due to those and other preventive measures, the number of people infected with seasonal influenza seems to remain extremely small. However, in a COVID-19 infected environment, the relationship among the mask wearing rate, outing rate and number of people infected with seasonal influenza was analyzed by principal component analysis (PCA) using a spot map in a three-dimensional space [17]. The analysis results showed that refraining from going out was far more effective in preventing seasonal influenza infection than wearing a mask [17]. The results of these analyses support that the WHO does not recommend wearing masks to prevent seasonal influenza infections.

Immigration restrictions for each country are indicated by the Travel Advisory Level or COVID-19 Travel Regulations. All countries worldwide, with respect to immigration, are either totally restrictive or partially restrictive. The number of new cases of the 2020 - 2021 seasonal influenza virus infections in the Northern Hemisphere and the number of new cases of the 2020 seasonal influenza virus infection in the Southern Hemisphere are extremely lower than the average number of new cases of seasonal influenza virus infection worldwide. Some countries in South America did not implement rules on wearing of facemasks, hand washing and social distancing or did not have strict immigration restrictions as precautionary measures against the spread of COVID-19. However, even in these countries, compared with the annual average number of new cases of seasonal influenza virus infection, the number of new cases of the 2020 seasonal influenza virus infections was extremely small. Most countries worldwide have strict immigration restrictions as a precautionary measure against the spread of COVID-19, so there is no movement of global people. As a result, even in countries where strict preventive measures against the spread of COVID-19 have not been taken, the number of new cases of seasonal influenza virus infection is considered extremely small.

Variants of the new coronavirus are spreading rapidly in the United Kingdom [18]. The British government has chosen warning the British people of "staying at home" as its advice. People's interactions were severely restricted during Christmas time in England, Scotland and Wales. Denmark, the Netherlands, Italy and Australia have banned travel from the United Kingdom with the aim of preventing the spread of a new COVID-19 variant. Much is still unknown about the strength, illness severity, or rate of population spread of this strain of COVID-19.

The Ministry of Foreign Affairs of Japan is collecting information to decide travel restrictions between the UK and Japan. Japan is different from the European Union (EU) region where it is relatively easy to move around or travel. In addition, the Ministry of Foreign Affairs of Japan is calling attention to Japanese people in the UK on the overseas safety website for variants of the new coronavirus. In mid-December 2020, Japan Airlines and All Nippon Airways were consid- ering specific responses given their frequent direct flights to London. The government of the world's countries must consider a preventive measure against the spread of the variants of the new coronavirus from a variety of angles.

\section{Conclusion}

The number of people infected with the 2020 seasonal influenza virus has also declined significantly in some South American countries where good policies are not being implemented to prevent COVID-19. Perhaps, severe restriction of immigration in each country stops the global transmission of seasonal influenza virus. As a result, the number of people infected with the 2020 - 2021 seasonal influenza virus in each country is considered to have decreased significantly.

\section{Footnotes}

Note 1. Brazilian President has downplayed COVID-19 as a "slight cold". As a result, Brazilian President opposes the suspension of operations required by state governments for commercial facilities. As a result, the Brazilian Ministry of Health announced that as of December 26, 2020, the number of SARS-CoV-2 infected people was 7,448,560 people and number of deaths from COVID-19 was 21,000 people. Meanwhile, compared to the same period of the year, the number of people infected with the 2020 seasonal influenza virus in Brazil has decreased by about $40 \%$, and the number of deaths due to COVID-19 has been halved. https://www.worldometers. info/coronavirus/country/brazil/; https:/www.economist.com/ graphic-detail/2020/09/12/the-southern-hemisphere-skippedflu-season-in-2020 [4].

Note 2. Apart from vaccination and antiviral treatment, the public health management includes personal protective measures like: 1) Regular hand washing with proper drying of the hands; 2) Good respiratory hygiene - covering mouth and nose when coughing or sneezing, using tissues and disposing of them correctly; 3) Early self-isolation of those feeling unwell, feverish and having other symptoms of influenza; 4) Avoiding close contact with sick people; 5) Avoiding touching one's eyes, nose or mouth (https:/www.who.int/news-room/factsheets/detail/influenza-(seasonal)).

\section{Acknowledgments}

We thank all the medical staffs and co-medical staffs for providing and helping medical research at National Hospital Organization Kyoto Medical Center. We appreciate Crimson Interactive Japan Co., Ltd for revising and polishing our manuscript by Native English.

\section{Financial Disclosure}

This clinical research was performed with the support of the 
following research funding: Japan Society for the Promotion of Science for TH (grant No. 19K09840), START-program Japan Science and Technology Agency (JST) for TH (grant No. STSC20001) and National Hospital Organization Multicenter Clinical Study for TH (grant No. 2019- Cancer in general-02).

\section{Conflict of Interest}

None to declare.

\section{Informed Consent}

Not applicable.

\section{Author Contributions}

TH performed most of the clinical medicine and coordinated the project. $\mathrm{TH}$ performed diagnostic pathological studies. $\mathrm{TH}$ created the study and wrote the manuscript. IK carefully reviewed the manuscript and commented on aspects of clinical medicine. IK shared information on clinical medicine and oversaw the entirety of the study.

\section{Data Availability}

The authors declare that data supporting the findings of this study are available within the article.

\section{References}

1. Olsen SJ, Azziz-Baumgartner E, Budd AP, Brammer L, Sullivan S, Pineda RF, Cohen C, et al. Decreased Influenza Activity During the COVID-19 Pandemic - United States, Australia, Chile, and South Africa, 2020. MMWR Morb Mortal Wkly Rep. 2020;69(37):1305-1309.

2. Nelson MI, Viboud C, Vincent AL, Culhane MR, Detmer SE, Wentworth DE, Rambaut A, et al. Global migration of influenza A viruses in swine. Nat Commun. 2015;6:6696.

3. Bedford T, Riley S, Barr IG, Broor S, Chadha M, Cox NJ, Daniels RS, et al. Global circulation patterns of seasonal influenza viruses vary with antigenic drift. Nature. 2015;523(7559):217-220.

4. Russell CA, Jones TC, Barr IG, Cox NJ, Garten RJ, Greg- ory $\mathrm{V}$, Gust ID, et al. The global circulation of seasonal influenza A (H3N2) viruses. Science. 2008;320(5874):340346.

5. Steffen R, Rickenbach M, Wilhelm U, Helminger A, Schar M. Health problems after travel to developing countries. J Infect Dis. 1987;156(1):84-91.

6. Steffen R, Lobel HO. The epidemiological basis for practice of travel medicine. J Wilderness Med. 1994;5:56-66.

7. Hirsch A, Creighton C. Handbook of geographical and historical pathology. London: The New Sydenham Society, 1883 .

8. Hope-Simpson RE. The role of season in the epidemiology of influenza. J Hyg (Lond). 1981;86(1):35-47.

9. Terajima M, Cruz J, Co MD, Lee JH, Kaur K, Wrammert J, Wilson PC, et al. Complement-dependent lysis of influenza a virus-infected cells by broadly cross-reactive human monoclonal antibodies. J Virol. 2011;85(24):1346313467.

10. COVID-19 Travel Regulations Map* (powered by Timatic) IATA - International Travel Document News (iatatravelcentre.com).

11. Influenza: Vaccination Still the Best Protection. https:// www.fda.gov/fdac/features/2006/506_influenza.html.

12. Key Facts about Influenza (Flu) Vaccine. https://www. cdc.gov/flu/protect/keyfacts.htm.

13. Archived disappears from flu preventive measures. The Wayback Machine. 2013. https://web.archive.org/ web/20130208180114/http:/cgi.daily-tohoku.co.jp/cgibin/news/2013/01/29/new13012908top.htm

14. Influenza prevention measures that are often forgotten. Make a mask, wash your hands often. Japan Ministry of Health, Labor and Welfare. November 9, 2012. https:// www.mhlw.go.jp/bunya/kenkou/kekkaku-kansenshou01/ dl/poster24.pdf.

15. Prime Minister's Office "Beware of winter infectious diseases! - Influenza \& norovirus special feature" January 31, 2013. http://www.kantei.go.jp/jp/headline/winter2012_kan sen.html.

16. Ministry of Health, Labor and Welfare's influenza prevention awareness "Gargling is unfounded". January 31, 2013.

17. Hayashi T, Yaegashi N, Konishi I. COVID-19 era, Preventive effect of no going out against co-infection of the seasonal influenza virus and SARS-CoV-2. medRxiv. Cold Spring Harbor on line published at October 3, 2020.

18. James Gallagher. New coronavirus variant: What do we know? New coronavirus variant: What do we know? BBC News. 